\title{
Construcción, validez y confiabilidad de la Escala de Resiliencia Docente
}

\section{Construction, validity and reliability of the Scale of Teacher Resilience}

\author{
José L. Guerra ${ }^{1 a}$ \\ ${ }^{1}$ Universidad San Ignacio de Loyola, Lima, Perú.

\begin{abstract}
${ }^{a}$ Magister en Educación con Mención en Evaluación y Acreditación de la Calidad de la Educación por la Universidad San Ignacio de Loyola. Actualmente se desempeña como Coordinador Académico en la Facultad de Educación de la misma Universidad.
\end{abstract}

Recibido: 06-02-13

Aprobado: $25-08-13$

\section{Correspondencia}

Email: propositosyrepresentaciones@usil.edu.pe

Notas

\section{Citar Como:}

Guerra, J. (2013). Construcción, validez y confiabilidad de la Escala de Resiliencia Docente. Propósitos y Representaciones, 1(1), 59-86. doi: http://dx.doi.org/10.20511/ pyr2013.v1n 1.7

El estudio que sirve de base al presente artículo fue patrocinado por el Gobierno Regional del Callao (Perú) y se desarrolló en el marco del Programa Académico de Maestría en Educación de la Universidad San Ignacio de Loyola. En la aplicación del instrumento se contó con la colaboraron las Magísteres Carmen Mundaca e Iris López.

(C) Universidad San Ignacio de Loyola, Vicerrectorado de Investigación y Desarrollo, 2013.

(cc) BY-NC-ND Este artículo se distribuye bajo licencia CC BY-NC-ND 4.0 Internacional (http://creativecommons.org/licenses/by-nc-nd/4.0/). 


\section{Resumen}

En la revisión que se ha realizado con relación a instrumentos para medir la resiliencia, no se ha encontrado ninguno construido específicamente desde y para docentes peruanos de las escuelas públicas de educación básica regular. Por tal motivo, uno de los objetivos del estudio que se informa fue construir un instrumento para medir la resiliencia, tomando los fundamentos teóricos expresados por Henderson y Milstein (2003). Para ello, participaron 710 docentes de ambos sexos pertenecientes a las escuelas públicas de la Región Callao. Los resultados muestran que 71 ítems se distribuyeron en cuatro factores: 1) participación significativa, 2) conducta prosocial, 3) autoestima y aprendizaje; y 4) percepción de apoyo. El coeficiente alfa de cronbach de la escala es de .957 , con lo cual se demuestra que la Escala de Resiliencia Docente (ER-D) es altamente confiable. Asimismo, la escala cuenta con validez de contenido apoyada por el análisis de diez jueces expertos y con validez de constructo. Es un instrumento probado en una muestra urbana y cuenta con baremos para la población chalaca.

Palabras clave: Resiliencia, docente, educación, medición.

\section{Summary}

Considering that there are no instruments to measure resilience specifically designed for peruvian teachers of elementary and secondary public schools, this study aimed to build an ad hoc instrument based on the theoretical foundations expressed by Henderson and Milstein (2003). We did this based on data from 710 public school teachers, both male and female of Callao region (Peru). The results show that 71 items concentrated around four factors: 1) significant participation, 2) prosocial behavior, 3) self-esteem and learning, and 4) perception of support. The cronbach's alpha coefficient is of .957 , which shows that the resilience scale is highly reliable. The scale has been validated by 10 expert judges.

Key words: Resilience, teacher, education, measurement. 
En el Perú de los noventa, los bajos rendimientos educativos demostraban tres cosas: que los estudiantes de primaria y de secundaria alcanzaban bajos niveles de logro en lenguaje y matemáticas; que en ello existían diferencias significativas entre escuelas estatales y privadas, y que esas brechas de aprendizaje ponían en la peor situación a los estudiantes de zonas rurales, más aún si eran de procedencia indígena.

La evaluación internacional realizada en 1997 por el Laboratorio Latinoamericano de Evaluación de la Calidad de la Educación (LLECE UNESCO, 1998) reveló que los estudiantes peruanos tenían un rendimiento por debajo del promedio de los países latinoamericanos que participaron en el estudio, ubicándose en el antepenúltimo lugar en lenguaje y último en matemáticas. Además, la prueba del Programa Internacional de Evaluación de Estudiantes (PISA), realizada en el 2001 en más de 40 países del mundo por la Organización para la Cooperación y el Desarrollo Económico (OCDE), con el propósito de evaluar el desempeño en aptitudes para la lectura, las matemáticas y las ciencias en jóvenes de 15 años de edad, ubicaron a los estudiantes peruanos en el último lugar de la lista (UMC, 2002).

Con estos resultados el sistema educativo peruano mostraba su fracaso en el campo de los aprendizajes básicos. Pese a los notables logros en cobertura escolar, los mayores niveles de asistencia de niños y niñas no aseguraron los aprendizajes que las escuelas les prometían.

Es así como los múltiples diagnósticos, que alarmaban sobre la crisis que afectaba a la educación nacional, obligaron a las autoridades a declarar la emergencia educativa en el Perú. Esta decisión tenía objetivos a lograr en el mediano y largo plazo: frenar y revertir el deterioro en la calidad de la educación; dar prioridad a la educación básica, pilar del resto de la educación; poner énfasis en la formación continua y actualización de los maestros; y, efectuar con carácter urgente la evaluación y reparación de aulas y colegios.

En este contexto se aprobaron una serie de leyes con el propósito de apuntar a la mejora educativa. Por ejemplo, la Ley General de Educación $\mathrm{N}^{\circ}$ 28044 que, entre otras cosas, establece que uno de los factores principales para el logro de la calidad educativa es una formación inicial y permanente que garantice la idoneidad de los docentes y autoridades educativas. 
Posteriormente, la Ley de la Carrera Pública Magisterial (CPM) No 29062, que establecía las condiciones de ascenso a los diversos niveles de la CPM, así como los criterios y procesos de evaluación para el ingreso y permanencia de docentes, determinando las bases de un sistema de formación continua de docentes integral, pertinente, intercultural y de calidad. Sin embargo, el actual gobierno ha derogado la ley $\mathrm{N}^{\circ} 29062$ y promulgado la Ley de Reforma Magisterial ํ29944 a finales del año 2012, con el propósito de incluir a todo el magisterio nacional en un mismo régimen legal, salarial, de evaluación y de permanencia en la carrera.

En concordancia con la Ley General de Educación, se promulgó la ley del Sistema Nacional de Evaluación, Acreditación y Certificación de la Calidad Educativa (SINEACE) No 28740, cuyo reglamento del 2007 establece la necesidad de que toda institución educativa, pública o privada, sea evaluada y acreditada. Ello implica promover una cultura de la evaluación y de la calidad, así como establecer estándares y mecanismos idóneos de aseguramiento de la calidad del servicio educativo.

Sin embargo, y a pesar de las reformas legales, la sociedad no ha logrado aún reivindicar al maestro. La pauperización de los salarios docentes los ha llevado a multiplicar espacios y tiempos de trabajo. No son pocos los maestros que se desempeñan en dos turnos y los profesores que recorren diariamente varias escuelas, tratando de sumar horas de trabajo cuya compensación salarial les permita enfrentar las necesidades básicas de sus familias. Por el contrario, la Ley de Reforma Magisterial, basada en un modelo económico que pretende modernizar la educación a través de la recompensa a la superación y el logro, promete al docente horizontes de mejora mediante la evaluación.

Por otro lado, la resistencia del gremio magisterial a la evaluación del desempeño docente, ha sido y sigue siendo una realidad. La ley $\mathrm{N}^{\circ} 29062$ indicaba que ser desaprobado tres veces en la evaluación de desempeño, o resistirse a ser evaluado en dos ocasiones, será causal de retiro de la CPM. El maestro enfrenta ahora una serie de mecanismos de capacitación y perfeccionamiento adoptados por el Estado. Esta situación altera la vida familiar y laboral para aquellos que deciden dedicar tiempo al estudio. Para aquellos que no lo hacen, les genera una situación inestable y de desventaja 
en los procesos de evaluación laboral. Sin embargo, hay una realidad que es peor aún, la mayoría de los docentes han realizado cursos sin que los mismos tengan un impacto positivo en su propia formación y en el proceso evaluativo.

A este punto, cabe agregar la situación social de violencia y criminalidad que vive el país, convirtiendo a la ciudad del Callao en uno de los lugares más inseguros a nivel nacional. En este contexto, surge la necesidad de reflexionar sobre el profesor con capacidades resilientes, ya que el docente de aula sigue siendo el actor insustituible del proceso educativo. Las buenas condiciones materiales de vida y de trabajo del maestro favorecen su desempeño docente, pero el éxito en el aprendizaje de los alumnos depende, en última instancia, de sus capacidades, sus cualidades, su formación y su buen criterio. Es por ello que se busca cambiar en el magisterio arraigadas prácticas docentes: desgano, falta de criterio, incapacidad para asumir responsabilidades frente a los bajos resultados de aprendizaje, resistencia al uso de materiales y textos educativos, tensión en la relación con los padres de familia, entre otras.

Es cierto que el presupuesto en educación ha crecido durante los últimos años y con ello las condiciones laborales han ido mejorando. Sin embargo, se puede observar que el aumento de la inversión económica en educación no genera automáticamente la reivindicación social tan anhelada y con ello la satisfacción en el desempeño de la docencia. Si no se consideran otros factores persistirán elementos como la falta de compromiso, de sentido de pertenencia, de solidaridad, de convivencia, entre otras carencias. En esta misma línea, nace la necesidad de seguir investigando y diseñando programas que promuevan y refuercen las características resilientes de los docentes y de las comunidades educativas. Los docentes "chalacos" están llamados a descubrir en su propio contexto educativo nuevos motivos para la superación $\mathrm{y}$ transformación personal y profesional.

El problema es complejo. Tratándose de un magisterio en crisis, surge la importancia de revalorar la profesión docente propiciando capacidades resilientes que favorezcan una actitud firme con relación a los procesos de evaluación y certificación. Este nuevo proceso que tiende a jerarquizar al docente, desde lo académico y profesional, demanda personas que puedan enfrentar con éxito los desafíos de la educación peruana. 
La situación de abandono material y psicológico en el que se encuentran miles de niños y adolescentes, hace que la escuela sea la única esperanza que muchos de ellos tienen para pasar de la situación de riesgo a la conducta resiliente. Por ello, es importante analizar la conducta resiliente en los ámbitos educativos. Henderson y Milstein (2003) encuentran que es poco realista pretender que los alumnos sean resilientes si sus docentes no lo son.

Como se puede entender, los docentes son de gran influencia en el desarrollo de las habilidades de resiliencia de los estudiantes, razón por la que ellos también deben ser resilientes. Pero las condiciones materiales y profesionales en las cuales trabajan, hacen que muchas veces les resulte difícil el desarrollo de esas habilidades. Se puede decir que la construcción de una educación de calidad y la reivindicación del docente son procesos conjuntos que necesitan de un mismo valor inicial: la resiliencia.

El presente artículo tiene como objetivo validar una Escala de Resiliencia Docente (ER-D) que permita establecer y reconocer los niveles en que esta característica se presenta en el magisterio chalaco. La escala ha sido elaborada a partir del estudio de otras experiencias sobre el tema, tanto nacionales como extranjeras.

Para Rirkin y Hoopman (1991), citados por Henderson y Milstein (2003), la resiliencia es la capacidad de recuperarse, sobreponerse y adaptarse con éxito frente a la adversidad, y de desarrollar competencia social, académica y vocacional, pese a estar expuesto a un estrés grave o simplemente a las tensiones inherentes al mundo de hoy. También sirve como modelo comprehensivo para fomentar el éxito académico y social de los alumnos, así como promover capacidades y motivación para enfrentar los desafíos de la educación actual, como lo sostienen también Henderson y Milstein (2003).

Los mismos autores señalan seis factores constructores de resiliencia en los docentes:

1. Enriquecer los vínculos. La vida profesional de los docentes transcurre casi por completo en compañía de sus alumnos, sin la posibilidad de interactuar regularmente con sus colegas. Inclusive, las evaluaciones de desempeño profesional de los docentes solo guardan relación con lo que 
éstos realizan en sus aulas, sin tener en cuenta el trabajo en equipo que permite vincularse a los otros docentes. Un primer aspecto será procurar que se dé una interacción del equipo de docentes, pues así se promueven las relaciones afectivas y la sensación de pertenecer a un equipo. En segundo lugar, la convocatoria al personal para que contribuyan a determinar los objetivos y las actividades de la escuela, puede hacerles sentir que pertenecen a algo más grande, que los involucra y, a la vez, incrementa las perspectivas de desarrollar programas educativos eficaces. También puede eliminar las barreras artificiales que suelen existir entre docentes y directivos, en tanto ambos grupos advierten la interconexión de sus roles en el logro de la excelencia educacional. En tercer lugar, las interacciones personales, como las tutorías y el trabajo en grupos pequeños, tienden a afianzar la sensación de pertenencia de los docentes al tiempo que incrementan la eficacia de la enseñanza.

2. Establecer límites claros y firmes. Los autores señalan que los docentes llevan a cabo sus actividades dentro de un complejo sistema de reglas (entrega de informes, horarios de salida, servicios requeridos, expectativas respecto de la disciplina de los alumnos), que a menudo son poco claras. Se piensa que la libertad, la creatividad y el crecimiento requieren pocas restricciones o ninguna. En realidad la creatividad y el crecimiento sólo son posibles con expectativas que no sean arbitrarias, caprichosas, desigualmente aplicadas o, lo que es aún peor, nulas. Los docentes se sienten seguros cuando tienen claros los límites dentro de los cuales se manejan. De allí la necesidad de que su creatividad no sea improvisada, sino que responda a un plan o proyecto que se articule con otros dentro de los objetivos y normas de la escuela.

3. Aprendizaje de habilidades para la vida. Los educadores necesitan desarrollarse profesionalmente para responder a los variados y crecientes desafíos que enfrentan. La rapidez con que surgen nuevos datos, avances tecnológicos y cambios sociales hace que estos mínimos conocimientos y habilidades resulten obsoletos. Para estos autores las escuelas pueden brindar oportunidades de desarrollo profesional significativas. Esto requiere que los docentes y directivos determinen cuáles son sus necesidades más apremiantes en materia de desarrollo profesional, en lugar que el personal del Ministerio de Educación (MINEDU) establezca los objetivos y el contenido de los cursos a aprender. También requiere 
que las jurisdicciones asignen recursos para esos fines. Además, la concepción estrecha de desarrollo profesional, normalmente referida a la mejora en el desempeño de la tarea docente, puede ampliarse de modo que incluya procesos interactivos tales como los de fijar metas, manejar conflictos, comunicarse y resolver problemas, es decir, las habilidades básicas que contribuyen a una mayor resiliencia.

4. Brindar afecto y apoyo. La principal recompensa de los educadores, como profesionales, radica en la satisfacción intrínseca de saber que se está cumpliendo una función importante. Necesitan recibir retroalimentación, de parte de los supervisores y pares, que les permita saber que están haciendo bien su trabajo, pues de lo contrario podrían interpretar el silencio como un indicio de fracaso.

La práctica de brindar esa retroalimentación adquiere especial relevancia debido al modo en que suelen distribuirse las recompensas extrínsecas, de por sí limitadas. Las políticas salariales y la relativa ausencia de fondos para otorgar reconocimientos especiales no propician la concesión de recompensas extrínsecas significativas a los desempeños individuales.

Es importante modificar el sistema de recompensas establecido. Un cambio posible sería introducir desafios profesionales y gratificaciones diferenciales para quienes los asuman. Podría implementarse un sistema de premios que reconozca los aportes sobresalientes, o bien sustituir la política salarial existente por otra que prevea recompensas diferenciales basadas en el esfuerzo y el efecto registrados. Estas sugerencias tal vez sean políticamente inquietantes. Gratificar los desempeños excepcionales e inducir a otros a tomar más iniciativas o perder su posición privilegiada puede ser justamente lo que se necesita.

5. Establecer y transmitir expectativas elevadas. Si los educadores reciben el mensaje de que su tarea primordial es mantener el orden y que solo se espera de ellos que lleguen a fin de año sin mayores contratiempos, tanto la excelencia educativa como la construcción de resiliencia quedan invalidadas. Si los docentes de alto rendimiento inspiran más animosidad que admiración a sus colegas, se debilitan las expectativas elevadas y la excelencia. 
Lo que motiva a los educadores es la convicción de estar al servicio de causas que van más allá de ellos mismos y de sus funciones concretas. Esto ocurre cuando comparten una misión en común y objetivos orientados a cumplirla a través de la comprensión y valoración de los aportes que cada uno hace para convertir en realidad la misión y los objetivos. En la práctica esto significa trabajar en equipo, compartir tareas, promover la diversificación de los roles y alentar a los individuos a contribuir en aspectos que exceden sus funciones específicas. Además, implica reducir las tareas que no guardan relación directa con la labor docente, como completar formularios de informes innecesarios, a fin de que los educadores puedan concentrarse en llevar a cabo sus obligaciones concretas.

Si el trabajo es relevante, ya sea que se relacione con el rol específico del individuo o con la eficacia global de la escuela, es necesario protegerlo. Las expectativas elevadas requieren que los docentes dediquen el mayor tiempo posible a su labor. Es decir, que se les conceda tiempo para realizar actividades directamente relacionadas con el aprendizaje de sus alumnos.

6. Brindar oportunidades de participación significativa. Muchos de los docentes tienen más que ofrecer a su escuela de lo que realmente involucra sus funciones específicas. La resiliencia se promueve cuando se les otorgan posibilidades de aportar sus habilidades y energía en el lugar de trabajo. Los educadores requieren de situaciones para aprender nuevas habilidades y participar en actividades estimulantes. Actualmente, la reestructuración y la mayor responsabilidad de cada establecimiento educativo podría otorgar a los docentes roles significativos dentro de la institución escolar.

Para aprovechar esta posibilidad hay varios aspectos a tener en cuenta. Henderson y Milstein (2003) señalan, en primer lugar, que la definición del rol docente debe comprender responsabilidades a nivel de toda la escuela. Los directivos escolares podrían actuar como facilitadores de los procesos institucionales, además de cumplir funciones de autoridad.

En segundo lugar, habría que conceder tiempo a los miembros del personal para que elaboren planes en conjunto y capacitarlos a fin de que empleen con eficacia ese tiempo. En tercer lugar, debe asegurarse que el tiempo dedicado a planificar rinda beneficios a largo plazo, como un currículo más significativo o una política disciplinaria más clara y efectiva, que motive a todos a participar. 
Por último, si bien es importante alentar la participación en toda la escuela, hay que mantener el tiempo que se destina a las tareas específicas de cada rol, pues caso contrario, el personal podría sentirse demasiado exigido.

Por otro lado, Ospina (2007) considera que la comprensión de la resiliencia es aún insuficiente. Trabajar en el desarrollo o perfeccionamiento de nuevos instrumentos para su medición, apoyados en una revisión apropiada de la literatura, puede ser un aporte importante para avanzar en este campo. Señala, además, que es necesario trabajar en la construcción de instrumentos válidos y confiables que permitan mejorar la comprensión del fenómeno resiliente. Por último, concluye que dentro del desarrollo de instrumentos de medición de la resiliencia, es necesario plantear con mayor relevancia la pregunta sobre cómo construir instrumentos sensibles a las diferencias de género, edad y aspectos socioculturales. La medición de la resiliencia es un proceso complejo que amerita un trabajo interdisciplinario. Un instrumento con capacidad para abordar el fenómeno resiliente, en sus diferentes dimensiones, podría constituir una oportunidad de desarrollo teórico importante en el tema y de avanzar en propuestas de intervención que involucren la promoción de la resiliencia.

Ante el contexto difícil y cambiante de la educación, y las adversidades que rodean al maestro, es necesario saber si se poseen las capacidades necesarias para vencerlas y salir adelante. Medir cómo los profesores van tejiendo su resiliencia, a partir de sus interacciones con los diferentes actores del sistema escolar (alumnos, padres de familia, docentes, directivos, administrativos, personal de servicio, autoridades, entre otros), se presenta como una tarea compleja. Los estudios sobre resiliencia en el ámbito educativo aún son escasos, pero han ido aumentando a medida que se descubre el potencial del concepto para explicar e intervenir ciertos fenómenos de nuestra realidad educativa y social.

\section{Método}

\section{Población y descripción de la muestra.}

El universo de investigación estuvo conformado por 5.940 docentes de los tres niveles de la Educación Básica Regular (inicial, primaria y secundaria) en los diferentes centros educativos estatales de la Región Callao, de acuerdo 
a los datos brindados por el MINEDU. La muestra estuvo conformada por 710 docentes, varones y mujeres, de centros educativos estatales de la región y pertenecientes a los tres niveles. Fueron seleccionados a través de un muestreo de tipo intencional, siendo distribuidos de manera proporcional según los atributos de nivel educativo y distrito de la institución educativa.

Debido a que uno de los objetivos fue la elaboración de una escala de resiliencia para docentes, es que se decidió utilizar una muestra que cumpla con los requisitos básicos para la realización de un análisis factorial. Por lo cual, la muestra utilizada fue superior a la muestra representativa requerida. $\mathrm{El} \mathrm{45 \%} \mathrm{de}$ los participantes fueron del nivel de secundaria (320 docentes), el $41 \%$ del nivel primario (291 docentes) y el 14\% del nivel de inicial (99 docentes).

Fueron cinco las personas encargadas de administrar las pruebas piloto de la ER-D a la muestra seleccionada. Se visitaron a los docentes no solamente en las escuelas donde laboran sino también en sus centros de estudio (programas de capacitación y posgrado). Se realizaron sin mayor contratiempo las coordinaciones necesarias previas a la aplicación del instrumento. En cambio, en el momento de la aplicación, se presentaron dificultades vinculadas a actitudes de desconfianza o de suspicacia por parte de los docentes, debido tal vez al momento político del país signado, en el tema educativo, por las evaluaciones del Programa Nacional de Formación y Capacitación Permanente (PRONAFCAP), y las evaluaciones para la CPM.

\section{Instrumento.}

La ER-D fue creada siguiendo las pautas establecidas para la construcción de un instrumento psicométrico. Las etapas que se siguió fueron las siguientes:

\section{Etapa I: Construcción y validación del instrumento.}

Para la construcción del instrumento se tomó como base los conceptos teóricos expresados por Henderson y Milstein (2003). El proceso se inició con la definición de la variable resiliencia, definida tal y como se presenta en la introducción. Las dimensiones con sus respectivos indicadores, que se señalan en la tabla 1, sirvieron para la redacción de los ítems. 
Tabla 1.

Matriz de definición operacional para resiliencia en docentes

\begin{tabular}{|c|c|}
\hline Di & dores \\
\hline $\begin{array}{l}\text { Enriquecimiento } \\
\text { de los vínculos } \\
\text { prosociales. }\end{array}$ & $\begin{array}{l}\text { - Busca oportunidades de interactuar con otros. } \\
\text { - Puede interactuar fácilmente con otros, aunque existan } \\
\text { diferencias de jerarquía. } \\
\text { - Participa en actividades cooperativas. }\end{array}$ \\
\hline $\begin{array}{l}\text { Fijació } \\
\text { claro }\end{array}$ & $\begin{array}{l}\text { - Comprende y acepta las políticas y las reglas. } \\
\text { - Interviene en la elaboración y la modificación de las políticas y } \\
\text { las reglas. }\end{array}$ \\
\hline $\begin{array}{l}\text { Aprendizaje de } \\
\text { habilidades para } \\
\text { la vida. }\end{array}$ & $\begin{array}{l}\text { - Toma parte en actividades de desarrollo profesional significativo. } \\
\text { - Tiene una elevada autoestima que es promovida por } \\
\text { oportunidades de aprendizaje adulto. } \\
\text { - Presta ayuda y la recibe de otros educadores. }\end{array}$ \\
\hline Afec & $\begin{array}{l}\text { - Tiene sensación de pertenencia a través de mensajes de } \\
\text { valoración y apoyo. } \\
\text { - Piensa que la comunidad respalda las actividades de los } \\
\text { educadores. } \\
\text { - Cree que los sistemas de recompensas alientan las iniciativas } \\
\text { individuales. }\end{array}$ \\
\hline
\end{tabular}

\begin{tabular}{cc}
\hline $\begin{array}{c}\text { Establecimiento } \\
\text { y transmisión } \\
\text { de expectativas } \\
\text { elevadas. }\end{array}$ & $\begin{array}{c}\text { - Demuestra confianza en su propio potencial de excelencia y en } \\
\text { el de los demás. }\end{array}$ \\
\hline & $\begin{array}{c}\text { - Se siente valorado en el desempeño de su rol. } \\
\text { sus expectativas laborales. }\end{array}$ \\
\hline $\begin{array}{c}\text { - Valora el aumento de responsabilidad del establecimiento } \\
\text { educativo como medio de asegurar la intervención del docente } \\
\text { en la adopción de decisiones. }\end{array}$ \\
$\begin{array}{c}\text { oportunidades } \\
\text { significativa. }\end{array}$ & $\begin{array}{l}\text { Dedica el tiempo y adquiere las habilidades requeridas para } \\
\text { participar con eficacia. }\end{array}$ \\
& $\begin{array}{c}\text { Está al tanto de lo que sucede y toma parte en la celebración de } \\
\text { los éxitos de la escuela. }\end{array}$
\end{tabular}

Tomado de Henderson y Milstein (2003).

Se redactaron un total de 75 ítems, que luego se llevaron a un formato de cuestionario para el proceso de validación por juicio de expertos. La tabla 2 presenta el número de ítems que corresponden a cada dimensión, siendo la dimensión de "oportunidades de participación significativa" la que mayor número de ítems tuvo en la primera versión de la escala, a diferencia de la dimensión de "fijación de límites claros y firmes", que tuvo menor número de ítems. 
Tabla 2.

Dimensiones y sus respectivos items en juicio de expertos

\begin{tabular}{lcc}
\hline \multicolumn{1}{c}{ Dimensiones } & Ítems & Total \\
\hline Enriquecimiento de los vínculos prosociales. & $1-13$ & 13 \\
Fijación de límites claros y firmes. & $14-21$ & 8 \\
Aprendizaje de habilidades para la vida. & $22-33$ & 12 \\
Afecto y apoyo. & $34-43$ & 10 \\
Establecimiento y transmisión de & $44-56$ & 13 \\
expectativas elevadas. & $57-75$ & 19 \\
Oportunidades de participación significativa. & & 75 \\
\hline \multicolumn{1}{c}{ Total } & \\
\hline
\end{tabular}

Para establecer la validez de contenido de la escala, se entregó el cuestionario a 10 profesionales (jueces) vinculados al tema de la resiliencia y especialistas en metodología de la investigación, ocho psicólogos y dos educadores. Se les consultó sobre el grado de adecuación de los ítems en torno al tema y se eliminaron aquellos ítems en que tres o más jueces tuviesen observaciones o reparos.

\section{Etapa II: Aplicación del instrumento.}

Para la aplicación del instrumento se encuestó a 10 sujetos por ítem (Morales, 2006), dando un total de 710 encuestados. Se corroboró el número proporcional de la muestra según nivel y distrito como se muestra en la tabla 3.

Tabla 3.

Número de docentes encuestados según nivel y distrito

\begin{tabular}{lcccc}
\hline \multirow{2}{*}{ Distrito } & \multicolumn{3}{c}{ Nivel } & Total \\
\cline { 2 - 4 } & Inicial & Primaria & Secundaria & \\
\hline Callao & 46 & 129 & 130 & 305 \\
La Perla & 6 & 14 & 16 & 36 \\
Bellavista & 8 & 18 & 40 & 66 \\
La Punta & 0 & 2 & 0 & 2 \\
Carmen de la Legua & 3 & 14 & 14 & 31 \\
Ventanilla & 36 & 114 & 120 & 270 \\
\hline \multicolumn{1}{c}{ Total } & 99 & 291 & 320 & 710 \\
\hline
\end{tabular}




\section{Etapa III: Confiabilidad del instrumento.}

El análisis factorial, con extracción de factores y reducción de ítems, se llevó a cabo luego de la aplicación. Los cuestionarios fueron numerados de acuerdo a la base de datos para tener un control y búsqueda rápida de las respuestas. El análisis permitió tomar la decisión de agrupar la observación en cuatro factores.

Finalmente se hizo un análisis psicométrico de correlación ítem-test corregida (ritc) y del coeficiente de confiabilidad por consistencias alfa de Cronbach (Muñiz, 1996), para establecer los niveles de consistencia interna de la escala.

\section{Resultados}

Para la validez de contenido se aplicó el coeficiente de Aiken (Escurra, 1988), quedando como resultado que de los 75 ítems iniciales se mantiene 71 para el instrumento de la prueba piloto. En la tabla 4 se observa que las seis dimensiones alcanzaron un coeficiente de validez muy alto, con valores entre .87 y .94 , que determinan que el $91 \%$ de los ítems de la prueba son válidos para la escala.

Tabla 4.

Validez de contenido de la ER-D

\begin{tabular}{ll}
\hline \multicolumn{1}{c}{ Dimensiones } & $\mathrm{V}$ \\
\hline Enriquecimiento de los vínculos prosociales. & .89 \\
Fijación de límites claros y firmes. & .87 \\
Aprendizaje de habilidades para la vida. & .87 \\
Afecto y apoyo. & .94 \\
Establecimiento y transmisión de expectativas elevadas. & .92 \\
Oportunidades de participación significativa. & .93 \\
\hline \multicolumn{1}{c}{ Total } & .91 \\
\hline
\end{tabular}

\section{Confección y reproducibilidad preliminar del instrumento.}

El instrumento piloto quedó constituido por 71 ítems y un nuevo orden en las preguntas. En la tabla 5 se observa las seis dimensiones con sus correspondientes ítems positivos y negativos, con mayoría de los primeros para evitar el problema de la aquiescencia. 
Tabla 5.

Dimensiones y sus respectivos items en ER-D piloto

\begin{tabular}{|c|c|c|c|}
\hline \multirow{2}{*}{ Dimensiones } & \multicolumn{2}{|c|}{ Ítems } & \multirow{2}{*}{$\begin{array}{l}\text { Total de } \\
\text { ítems }\end{array}$} \\
\hline & Positivos & Negativos & \\
\hline $\begin{array}{l}\text { Enriquecimiento de los } \\
\text { vínculos prosociales. }\end{array}$ & $\begin{array}{c}7,13,25,37,55,61 \\
65\end{array}$ & $1,19,31,43,49$ & 12 \\
\hline $\begin{array}{l}\text { Fijación de límites } \\
\text { claros y firmes. }\end{array}$ & $2,8,20,32,38$ & 14,26 & 7 \\
\hline $\begin{array}{l}\text { Aprendizaje de } \\
\text { habilidades para la vida. }\end{array}$ & $\begin{array}{c}3,9,15,21,27,33 \\
39,45\end{array}$ & 51,57 & 10 \\
\hline Afecto y apoyo. & $4,28,40,46,58$ & $16,10,22,34,52$ & 10 \\
\hline $\begin{array}{l}\text { Establecimiento } \\
\text { y transmisión de } \\
\text { expectativas elevadas. }\end{array}$ & $\begin{array}{c}11,23,29,35,41 \\
59,69\end{array}$ & $\begin{array}{c}5,17,47,53,63, \\
67,\end{array}$ & 13 \\
\hline $\begin{array}{l}\text { Oportunidades } \\
\text { de participación } \\
\text { significativa. }\end{array}$ & $\begin{array}{c}12,18,30,36,42 \\
44,54,56,62,68 \\
70,71\end{array}$ & $\begin{array}{c}6,24,48,50,60 \\
64,66\end{array}$ & 19 \\
\hline Total & 44 & 27 & 71 \\
\hline
\end{tabular}

Los resultados alcanzados en el análisis factorial exploratorio (véase tabla 6), realizado a través del método de los componentes principales y el método de rotación Oblimin, establecen que la medida de adecuación del muestreo de Kaiser-Meyer-Olkin alcanzó un valor de .962, considerado adecuado. Por otro lado, el test de esfericidad de Bartlett presenta un valor que es significativo $(B=22169.096 ; p<.000)$. Ello indica que los coeficientes de correlación entre los ítems fueron lo suficientemente elevados como para continuar con el análisis factorial.

Tabla 6.

Validez de constructo a través del análisis factorial exploratorio de la ER-D

KMO y Prueba de Bartlett

Medida de Adecuación Muestral de Kaiser-Meyer-Olkin

.962

Chi-Cuadrado

aproximado

22169.096

Prueba de Esfericidad de Barlett

Gl

2485

Sig.

.000 
Los resultados del análisis indican que cuatro factores permiten explicar el $40.43 \%$ de la varianza total. El primer factor explicó el $19.68 \%$ de la varianza total de los ítems que corresponden al área de participación significativa. El segundo factor explicó el $11.78 \%$ en el área de conducta prosocial. El tercero el $4.61 \%$ de autoestima y aprendizaje. Mientras el cuarto el $4.35 \%$ del área de percepción y apoyo. Los resultados alcanzados permitieron concluir que la ER-D presenta validez de constructo (véase tabla 7).

\section{Tabla 7.}

Factorización de ejes principales de la ER-D

\begin{tabular}{|c|c|c|c|c|c|c|c|c|c|}
\hline \multirow{2}{*}{$\begin{array}{l}\text { Compo } \\
\text { nente }\end{array}$} & \multicolumn{3}{|c|}{ Autovalores iniciales } & \multicolumn{3}{|c|}{$\begin{array}{l}\text { Sumas de las saturaciones al } \\
\text { cuadrado de la extracción }\end{array}$} & \multicolumn{3}{|c|}{$\begin{array}{l}\text { Suma de las saturaciones al } \\
\text { cuadrado de la rotación }\end{array}$} \\
\hline & Total & $\begin{array}{l}\% \text { de la } \\
\text { varianza }\end{array}$ & $\begin{array}{c}\% \\
\text { acumulado }\end{array}$ & Total & $\begin{array}{l}\% \text { de la } \\
\text { varianza }\end{array}$ & $\begin{array}{c}\% \\
\text { acumulado }\end{array}$ & Total & $\begin{array}{l}\% \text { de la } \\
\text { varianza }\end{array}$ & $\begin{array}{c}\% \\
\text { acumulado }\end{array}$ \\
\hline 1 & 20.170 & 28.408 & 28.408 & 20.170 & 28.408 & 28.408 & 13.973 & 19.680 & 19.680 \\
\hline 2 & 3.794 & 5.343 & 33.751 & 3.794 & 5.343 & 33.751 & 8.367 & 11.784 & 31.465 \\
\hline 3 & 2.420 & 3.408 & 37.159 & 2.420 & 3.408 & 37.159 & 3.275 & 4.612 & 36.077 \\
\hline 4 & 2.325 & 3.275 & 40.434 & 2.325 & 3.275 & 40.434 & 3.093 & 4.357 & 40.434 \\
\hline 5 & 1.698 & 2.391 & 42.825 & & & & & & \\
\hline 6 & 1.386 & 1.952 & 44.777 & & & & & & \\
\hline 7 & 1.277 & 1.798 & 46.575 & & & & & & \\
\hline 8 & 1.186 & 1.670 & 48.245 & & & & & & \\
\hline 9 & 1.150 & 1.620 & 49.865 & & & & & & \\
\hline 10 & 1.103 & 1.554 & 51.419 & & & & & & \\
\hline 11 & 1.073 & 1.511 & 52.930 & & & & & & \\
\hline 12 & 1.015 & 1.430 & 54.359 & & & & & & \\
\hline
\end{tabular}

A continuación, en la tabla 8 se puede observar la carga factorial para la matriz de componentes principales de los cuatro factores de la ER-D. 
Tabla 8 .

Matriz de componentes principales de la ER-D

\begin{tabular}{|c|c|c|c|c|c|c|c|c|c|}
\hline \multirow{2}{*}{ Items } & \multicolumn{4}{|c|}{ Componente / Factor } & \multirow{2}{*}{ Items } & \multicolumn{4}{|c|}{ Componente / Factor } \\
\hline & 1 & 2 & 3 & 4 & & 1 & 2 & 3 & 4 \\
\hline 01 & .342 & .280 & .050 & -.106 & 37 & .360 & -.090 & -.154 & .149 \\
\hline 02 & .507 & -.007 & -.138 & .227 & 38 & .620 & -.182 & -.056 & -.126 \\
\hline 03 & .506 & -.036 & .065 & .018 & 39 & .606 & -.048 & -.047 & .104 \\
\hline 04 & .417 & -.181 & .361 & .169 & 40 & .728 & -.262 & -.074 & -.091 \\
\hline 05 & .399 & .357 & -.148 & -.032 & 41 & .467 & -.114 & .320 & .410 \\
\hline 06 & .397 & .004 & .107 & -.202 & 42 & .677 & -.140 & -.015 & -.011 \\
\hline 07 & .662 & -.077 & -.139 & -.053 & 43 & .239 & .360 & .006 & -.246 \\
\hline 08 & .632 & -.170 & -.101 & .026 & 44 & .647 & .031 & -.163 & .017 \\
\hline 09 & .512 & -.193 & .016 & -.069 & 45 & .682 & -.212 & -.052 & .012 \\
\hline 10 & .352 & .138 & .388 & .023 & 46 & .280 & -.160 & .473 & .374 \\
\hline 11 & .536 & -.215 & .182 & -.010 & 47 & .273 & .456 & .245 & .189 \\
\hline 12 & .600 & -.269 & .153 & -.137 & 48 & .594 & .061 & .034 & -.373 \\
\hline 13 & .621 & -.097 & -.125 & .039 & 49 & .384 & .298 & .009 & -.048 \\
\hline 14 & .547 & .386 & -.146 & .123 & 50 & .572 & .240 & .050 & -.143 \\
\hline 15 & .700 & -.036 & -.016 & -.019 & 51 & .540 & .282 & .013 & -.251 \\
\hline 16 & .562 & .374 & -.023 & .089 & 52 & .405 & .021 & .091 & -.384 \\
\hline 17 & .582 & .398 & -.079 & -.020 & 53 & .345 & .321 & .395 & .290 \\
\hline 18 & .497 & .009 & .061 & .131 & 54 & .400 & .229 & -.279 & .216 \\
\hline 19 & .380 & .293 & .042 & .091 & 55 & .743 & -.286 & -.012 & -.181 \\
\hline 20 & .585 & -.071 & .036 & .082 & 56 & .689 & -.324 & .038 & -.176 \\
\hline 21 & .542 & .148 & -.222 & .326 & 57 & .561 & .348 & .041 & -.263 \\
\hline 22 & .444 & .231 & .237 & -.051 & 58 & .077 & -.174 & .390 & .297 \\
\hline 23 & .201 & .171 & -.098 & .458 & 59 & .144 & -.337 & .407 & .014 \\
\hline 24 & .574 & .271 & .069 & -.117 & 60 & .450 & .268 & .030 & -.044 \\
\hline 25 & .714 & -.025 & -.079 & .034 & 61 & 699 & -.053 & -.053 & .004 \\
\hline 26 & .413 & .214 & .190 & -.106 & 62 & .708 & -.242 & .003 & -.005 \\
\hline 27 & .700 & -.185 & -.183 & .080 & 63 & .101 & .142 & .379 & -.324 \\
\hline 28 & .675 & -.219 & -.100 & -.033 & 64 & .501 & .424 & .007 & -.057 \\
\hline 29 & .593 & -.292 & .159 & .138 & 65 & .538 & -.441 & .119 & -.103 \\
\hline 30 & .663 & .015 & .003 & .184 & 66 & .612 & .330 & -.110 & -.167 \\
\hline 31 & .518 & .258 & .019 & .005 & 67 & -.123 & .333 & .276 & .072 \\
\hline 32 & .705 & -.101 & -.079 & .058 & 68 & .647 & -.335 & -.016 & -.195 \\
\hline 33 & .575 & .003 & -.186 & .149 & 69 & .130 & -.036 & -.332 & .416 \\
\hline 34 & .247 & .170 & .435 & .178 & 70 & .289 & .060 & -.277 & .272 \\
\hline 35 & .589 & .064 & -.142 & .117 & 71 & .716 & -.183 & -.086 & -.018 \\
\hline 36 & .745 & -.067 & .007 & -.066 & & & & & \\
\hline
\end{tabular}


En la Tabla 9 se presenta las cargas factoriales de la ER-D, con una rotación octogonal varimax.

Tabla 9.

Matriz de componentes rotados

\begin{tabular}{|c|c|c|c|c|c|c|c|c|c|}
\hline \multirow{2}{*}{ Items } & \multicolumn{4}{|c|}{ Componente / Factor } & \multirow{2}{*}{ Items } & \multicolumn{4}{|c|}{ Componente / Factor } \\
\hline & 1 & 2 & 3 & 4 & & 1 & 2 & 3 & 4 \\
\hline 01 & .125 & .438 & .006 & .040 & 37 & .324 & .071 & .270 & .028 \\
\hline 02 & .376 & .198 & .368 & .110 & 38 & .624 & .212 & .044 & .017 \\
\hline 03 & .412 & .242 & .086 & .164 & 39 & .492 & .255 & .236 & .139 \\
\hline 04 & .367 & .061 & -.003 & .477 & 40 & .751 & .196 & .094 & .045 \\
\hline 05 & .130 & .498 & .200 & -.074 & 41 & .327 & .078 & .230 & .579 \\
\hline 06 & .339 & .274 & -.129 & .058 & 42 & .619 & .250 & .130 & .121 \\
\hline 07 & .591 & .295 & .173 & -.008 & 43 & .028 & .479 & -.090 & -.093 \\
\hline 08 & .603 & .187 & .193 & .063 & 44 & .507 & .354 & .253 & .003 \\
\hline 09 & .527 & .137 & .023 & .085 & 45 & .664 & .187 & .159 & .107 \\
\hline 10 & .158 & .322 & -.100 & .395 & 46 & .198 & -.038 & .068 & .650 \\
\hline 11 & .531 & .130 & -.021 & .258 & 47 & -.105 & .484 & .139 & .338 \\
\hline 12 & .640 & .150 & -.099 & .182 & 48 & .501 & .462 & -.170 & -.056 \\
\hline 13 & .552 & .235 & .225 & .046 & 49 & .141 & .457 & .086 & .046 \\
\hline 14 & .202 & .561 & .357 & .039 & 50 & .336 & .538 & .022 & .069 \\
\hline 15 & .579 & .349 & .145 & .117 & 51 & .311 & .579 & -.042 & -.028 \\
\hline 16 & .215 & .577 & .263 & .125 & 52 & .371 & .336 & -.259 & -.051 \\
\hline 17 & .243 & .630 & .216 & .024 & 53 & -.004 & .398 & .131 & .535 \\
\hline 18 & .357 & .244 & .181 & .218 & 54 & .172 & .323 & .448 & -.043 \\
\hline 19 & .111 & .419 & .176 & .147 & 55 & .787 & .211 & -.011 & .052 \\
\hline 20 & .485 & .237 & .165 & .193 & 56 & .760 & .155 & -.053 & .087 \\
\hline 21 & .304 & .311 & .523 & .095 & 57 & .289 & .648 & -.053 & -.010 \\
\hline 22 & .205 & .453 & -.039 & .246 & 58 & .068 & -.144 & .006 & .501 \\
\hline 23 & -.015 & .123 & .484 & .199 & 59 & .266 & -.168 & -.234 & .383 \\
\hline 24 & .314 & .559 & .038 & .097 & 60 & .207 & .469 & .089 & .080 \\
\hline 25 & .580 & .347 & .227 & .096 & 61 & .587 & .326 & .181 & .099 \\
\hline 26 & .205 & .433 & -.066 & .173 & 62 & .700 & .185 & .117 & .150 \\
\hline 27 & .663 & .192 & .294 & .039 & 63 & .022 & .277 & -.423 & .151 \\
\hline 28 & .675 & .186 & .148 & .043 & 64 & .163 & .624 & .126 & .058 \\
\hline 29 & .595 & .059 & .109 & .333 & 65 & .686 & -.033 & -.092 & .166 \\
\hline 30 & .481 & .321 & .292 & .232 & 66 & .336 & .626 & .116 & -.072 \\
\hline 31 & .259 & .484 & .147 & .111 & 67 & -.328 & .204 & -.076 & .228 \\
\hline 32 & .613 & .275 & .232 & .110 & 68 & .743 & .124 & -.049 & .024 \\
\hline 33 & .443 & .258 & .350 & .041 & 69 & .079 & -.088 & .536 & -.022 \\
\hline 34 & .022 & .256 & -.023 & .494 & 70 & .170 & .114 & .442 & -.027 \\
\hline 35 & .421 & .326 & .312 & .061 & 71 & .683 & .233 & .167 & .069 \\
\hline 36 & .640 & .361 & .100 & .121 & & & & & \\
\hline
\end{tabular}


Tabla 10.

Correlación Ítem - escala total y alfa de Cronbach si se elimina el elemento

\begin{tabular}{|c|c|c|c|c|c|c|c|c|c|c|c|}
\hline \multicolumn{3}{|c|}{$\begin{array}{l}\text { Participación } \\
\text { significativa }\end{array}$} & \multicolumn{3}{|c|}{ Conducta prosocial } & \multicolumn{3}{|c|}{$\begin{array}{l}\text { Autoestima y } \\
\text { aprendizaje }\end{array}$} & \multicolumn{3}{|c|}{ Percepción de apoyo } \\
\hline Items & $\begin{array}{l}\text { Correlación } \\
\text { elemento- } \\
\text { total } \\
\text { corregida }\end{array}$ & $\begin{array}{l}\text { Alfa de } \\
\text { Cronbach } \\
\text { si se } \\
\text { elimina el } \\
\text { elemento }\end{array}$ & Items & $\begin{array}{l}\text { Correlación } \\
\text { elemento- } \\
\text { total } \\
\text { corregida }\end{array}$ & $\begin{array}{l}\text { Alfa de } \\
\text { Cronbach } \\
\text { si se } \\
\text { elimina el } \\
\text { elemento }\end{array}$ & Items & $\begin{array}{l}\text { Correlación } \\
\text { elemento- } \\
\text { total } \\
\text { corregida }\end{array}$ & $\begin{array}{l}\text { Alfa de } \\
\text { Cronbach } \\
\text { si se } \\
\text { elimina el } \\
\text { elemento }\end{array}$ & Items & $\begin{array}{l}\text { Correlación } \\
\text { elemento- } \\
\text { total } \\
\text { corregida }\end{array}$ & $\begin{array}{l}\text { Alfa de } \\
\text { Cronbach } \\
\text { si se } \\
\text { elimina el } \\
\text { elemento }\end{array}$ \\
\hline 2 & .476 & .954 & 1 & .346 & .955 & 21 & .513 & .954 & 4 & .410 & .954 \\
\hline 3 & .482 & .954 & 5 & .389 & .954 & 23 & .198 & .955 & 10 & .368 & .955 \\
\hline 6 & .383 & .954 & 14 & .536 & .954 & 54 & .374 & .954 & 34 & .268 & .955 \\
\hline 7 & .618 & .954 & 16 & .561 & .954 & 63 & .115 & .955 & 41 & .462 & .954 \\
\hline 8 & .587 & .954 & 17 & .572 & .954 & 69 & .095 & .955 & 46 & .286 & .955 \\
\hline 9 & .480 & .954 & 19 & .382 & .955 & 70 & .261 & .955 & 53 & .375 & .954 \\
\hline 11 & .512 & .954 & 22 & .451 & .954 & & & & 58 & .089 & .956 \\
\hline 12 & .567 & .954 & 24 & .567 & .954 & & & & 59 & .140 & .955 \\
\hline 13 & .584 & .954 & 26 & .412 & .954 & & & & & & \\
\hline 15 & .671 & .953 & 31 & .508 & .954 & & & & & & \\
\hline 18 & .480 & .954 & 43 & .242 & .955 & & & & & & \\
\hline 20 & .559 & .954 & 47 & .301 & .955 & & & & & & \\
\hline 25 & .681 & .953 & 49 & .382 & .955 & & & & & & \\
\hline 27 & .652 & .954 & 50 & .563 & .954 & & & & & & \\
\hline 28 & .626 & .954 & 51 & .529 & .954 & & & & & & \\
\hline 29 & .561 & .954 & 57 & .559 & .954 & & & & & & \\
\hline 30 & .641 & .954 & 60 & .448 & .954 & & & & & & \\
\hline 32 & .669 & .954 & 64 & .503 & .954 & & & & & & \\
\hline 33 & .537 & .954 & 66 & .597 & .954 & & & & & & \\
\hline 35 & .559 & .954 & & & & & & & & & \\
\hline 36 & .712 & .953 & & & & & & & & & \\
\hline 37 & .330 & .955 & & & & & & & & & \\
\hline 38 & .579 & .954 & & & & & & & & & \\
\hline 39 & .579 & .954 & & & & & & & & & \\
\hline 40 & .681 & .953 & & & & & & & & & \\
\hline 42 & .642 & .954 & & & & & & & & & \\
\hline 44 & .611 & .954 & & & & & & & & & \\
\hline 45 & .643 & .954 & & & & & & & & & \\
\hline 48 & .569 & .954 & & & & & & & & & \\
\hline 52 & .383 & .955 & & & & & & & & & \\
\hline 55 & .695 & .953 & & & & & & & & & \\
\hline 56 & .647 & .953 & & & & & & & & & \\
\hline 61 & .662 & .954 & & & & & & & & & \\
\hline 62 & .668 & .954 & & & & & & & & & \\
\hline 65 & .493 & .954 & & & & & & & & & \\
\hline 67 & -.093 & .957 & & & & & & & & & \\
\hline 68 & .598 & .954 & & & & & & & & & \\
\hline 71 & .677 & .953 & & & & & & & & & \\
\hline
\end{tabular}


Por otro lado, en relación a la consistencia interna de la escala se encontró que en los cuatro factores las correlaciones ítem-test, corregidas para cada valor, superan el criterio establecido por Kline (1993) de .20. Además se alcanzaron coeficientes alfa de Cronbach de .956 para el primer factor, así como de $.88, .56$ y .69 para los restantes.

Además, al realizar el análisis psicométrico de la escala total (véase la tabla 10), se alcanzaron valores de correlación ítem-test superiores al .20, a excepción de los ítems 23,58, 59 y 69. Sin embargo, se optó por mantenerlos en la medida que cuentan con valores superiores en el análisis por factores. El coeficiente alfa de Cronbach de la escala total es de .95, que demuestra que la ER-D es un instrumento altamente confiable.

Los ítems eliminados luego del análisis psicométrico (análisis factorial y correlación ítem-test corregida) fueron dos, 63 y 67.

\section{Factores del constructo.}

A la luz de los análisis realizados se definió el constructo resiliencia mediante cuatro factores importantes en el contexto escolar. Ellos son:

\section{Participación significativa.}

Convertirse en personas significativas, capaces de ser fuente real de apoyo para otros, pasa por actuaciones como: participar en actividades cooperativas; intervenir en la elaboración y la modificación de las políticas y las reglas; sentirse valorado en el desempeño de su rol; valorar el aumento de responsabilidad del establecimiento educativo, como medio de asegurar la intervención del docente en la adopción de decisiones; dedicar el tiempo y adquirir las habilidades requeridas para participar con eficacia; estar al tanto de lo que sucede y tomar parte en los éxitos de la escuela.

El trabajo coordinado, en equipo, entre los profesores, además de todas las ventajas que conlleva desde el punto de vista de la calidad de la intervención, genera en la escuela y en el mismo docente un gratificante efecto de seguridad. Este se ofrece como modelo de relaciones humanas, en el que las personas, más allá de las diferencias de cualquier tipo, pueden 
entenderse y apoyarse mutuamente. En este factor, el docente crea espacios significativos que facilitan el encuentro, en los que no hay límites. Entiende que necesita espacios de los que apropiarse, que desarrollen su protagonismo y sentido de pertenencia.

Este primer factor está representado por los siguientes ítems positivos: $2,3,7,8,9,11,12,13,15,18,20,25,27,28,29,30,32,33,35,36,37,38$, $39,40,42,44,45,55,56,61,62,64,66,69$. También por los siguientes ítems negativos: $6,48,49$ y 52 .

\section{Conducta prosocial.}

Se entiende por conducta prosocial a toda conducta social positiva con o sin motivación altruista. Positiva significa que no daña, que no es agresiva. A su vez, se entiende por motivación altruista el deseo de favorecer al otro con independencia del propio beneficio. No basta con preocuparse por el bienestar de los otros o con elegirlos como destinatarios del trabajo educativo, es necesario que se sientan queridos, elegidos y dignos de respeto.

Esta área, concretamente, mide si el docente busca oportunidades de interactuar con otros, si presta ayuda y la recibe de otros educadores; si tiene sensación de pertenencia a través de mensajes de valoración y apoyo; si demuestra confianza en su propio potencial de excelencia y en el de los demás.

Este segundo factor está representado por el ítem positivo 4 y los ítems negativos: $1,5,14,17,19,22,24,26,31,43,47,50,51,57,60$, 63 y 65.

\section{Autoestima y aprendizaje.}

En este factor el docente manifiesta tener una elevada autoestima, promovida por oportunidades de aprendizaje. La autoestima es el puente necesario entre el hecho de ser amado y la capacidad de amar, porque la experiencia de querer y ayudar a los otros supone la convicción de que todo ser humano es digno de ello, empezando por uno mismo. La autoestima y el aprendizaje de competencias personales, especialmente las habilidades vinculadas con la interacción con los otros, son factores constructores de resiliencia, que en 
la práctica educativa se pueden potenciar con una buena opinión del propio desempeño profesional o dedicar el tiempo suficiente al aprendizaje.

Este tercer factor está representado por los ítems positivos 21, 23, 67, 54 y 68 .

\section{Percepción de apoyo.}

Esta área mide si el docente piensa que la comunidad respalda las actividades de los educadores y si se siente respaldado por las autoridades en el cumplimiento de sus expectativas laborales. El objetivo último de este reconocimiento y valoración es que los docentes lleguen a un convencimiento realista acerca de sus potencialidades. Es importante que los docentes asuman la responsabilidad de los aspectos positivos de su práctica. Esto pasa por revalorar la enseñanza pública, apoyar los proyectos de innovación educativa, así como reconocer y gratificar la excelencia docente.

Este cuarto factor está representado por los siguientes ítems positivos: $41,46,58,59$. También por los ítems negativos 10, 16, 34 y 53.

Los cuatro factores resilientes encontrados en los docentes de la Región Callao, se derivan de las seis dimensiones de la rueda de la resiliencia descritas en la literatura. El estudio apunta a una confirmación de que el proceso resiliente en el docente incorpora las seis dimensiones descritas sobre la resiliencia en la escuela. El formato final de la ER-D se muestra en el Apéndice A.

En la tabla 11 se presentan los baremos de la prueba en la que se tomó en consideración la muestra total de los docentes evaluados. Se ofrece indicadores de resiliencia tanto por factores como por el total de la prueba. 
Tabla 11.

Rangos percentilares de la ER-D, baremos

\begin{tabular}{|c|c|c|c|c|c|c|}
\hline Pc & $\begin{array}{l}\text { Participación } \\
\text { significativa }\end{array}$ & $\begin{array}{l}\text { Conducta } \\
\text { prosocial }\end{array}$ & $\begin{array}{l}\text { Autoestima y } \\
\text { aprendizaje }\end{array}$ & $\begin{array}{c}\text { Percepción } \\
\text { de apoyo }\end{array}$ & TOTAL & Pc \\
\hline 1 & - a 86 & - a 42 & - a 13 & - a 13 & - a 170 & 1 \\
\hline 3 & $87-97$ & $43-45$ & $14-15$ & 14 & $171-185$ & 3 \\
\hline 5 & $98-100$ & $46-47$ & & $15-16$ & $186-195$ & 5 \\
\hline 7 & $101-103$ & $48-50$ & 16 & & $196-205$ & 7 \\
\hline 10 & $104-106$ & $51-53$ & 17 & 17 & $206-213$ & 10 \\
\hline 15 & $107-110$ & $54-59$ & & 18 & $214-220$ & 15 \\
\hline 20 & $111-115$ & $60-64$ & 18 & 19 & $221-223$ & 20 \\
\hline 25 & $116-123$ & $65-66$ & & 20 & $224-230$ & 25 \\
\hline 30 & $124-131$ & $67-68$ & 19 & 21 & $231-237$ & 30 \\
\hline 35 & $132-139$ & 69 & & 22 & $238-247$ & 35 \\
\hline 40 & $140-142$ & 70 & & 23 & $248-256$ & 40 \\
\hline 45 & $143-145$ & $71-72$ & 20 & & $257-263$ & 45 \\
\hline 50 & $146-150$ & $73-76$ & 21 & $24-26$ & $264-272$ & 50 \\
\hline 55 & $151-152$ & 77 & 22 & & $273-276$ & 55 \\
\hline 60 & $153-155$ & 78 & & 27 & $277-281$ & 60 \\
\hline 65 & $156-157$ & $79-81$ & & 28 & $282-285$ & 65 \\
\hline 70 & $158-160$ & $82-83$ & 23 & 29 & $286-290$ & 70 \\
\hline 75 & $161-163$ & 84 & & 30 & $291-296$ & 75 \\
\hline 80 & $164-166$ & $85-87$ & & 31 & 297 - 302 & 80 \\
\hline 85 & $167-172$ & $88-90$ & 24 & 32 & $303-308$ & 85 \\
\hline 90 & $173-174$ & 91 & & 33 & $309-313$ & 90 \\
\hline 93 & $175-178$ & & & & $314-317$ & 93 \\
\hline 95 & 179 & 92 & 25 & $34-35$ & $318-323$ & 95 \\
\hline 97 & $180-182$ & $93-94$ & & 36 & $324-329$ & 97 \\
\hline 99 & $183-185$ & 95 & & $37-40$ & $330-345$ & 99 \\
\hline Media & 142.69 & 73.15 & 20.76 & 25.11 & 261.73 & Media \\
\hline Mediana & 149 & 74 & 21 & 25 & 269 & Mediana \\
\hline $\begin{array}{l}\text { Desv. } \\
\text { típ. }\end{array}$ & 24.55 & 12.92 & 2.62 & 5.53 & 38.66 & $\begin{array}{l}\text { Desv. } \\
\text { típ. }\end{array}$ \\
\hline Suj. & 710 & 710 & 710 & 710 & 710 & Suj. \\
\hline
\end{tabular}




\section{Conclusiones}

- El coeficiente alfa de Cronbach de la ER-D total es de .957, con lo cual se demuestra que es un instrumento altamente confiable. Dicho instrumento posee una base teórica sólida, a saber, el modelo de Henderson y Milstein (2003).

- La ER-D cuenta con validez de contenido, apoyada por el análisis de 10 jueces conocedores de la materia y con experiencia de investigación en el campo. La opinión de los jueces expertos comprueba que la escala explora la capacidad de resiliencia en los docentes y, por lo tanto, es válida en 71 de sus 75 ítems.

- El instrumento diseñado cuenta con validez de constructo. Es una escala con cuatro factores y 69 ítems; un puntaje máximo de 345; con opciones de respuesta tipo Likert; con un tiempo estimado de respuesta de 20 minutos; y fue diseñada para ser auto administrada. $\mathrm{Su}$ formato es de bajo costo, su tabulación resulta sencilla y de fácil vaciado en una base de datos.

- La ER-D es un instrumento probado en una muestra urbana y cuenta con baremos para la población "chalaca".

- La ER-D permite obtener puntajes por factores. Esta característica permitirá intervenir puntualmente en las áreas más débiles y poder apoyarse en los puntos fuertes del docente observado.

- Es recomendable continuar profundizando en el marco teórico. El paradigma de la resiliencia en la escuela se presenta como un espacio interdisciplinario de confluencia de esfuerzos profesionales procedentes de ámbitos diversos: educación, psicología, sociología, antropología, administración, política, entre otros.

- Por otro lado, se propone como parte de esta línea de investigación, evaluar la sensibilidad al cambio de la ER-D, mediante su aplicación después de un tiempo determinado. El propósito sería realizar un seguimiento más adecuado y dinámico, evaluando si la mejora o disminución de los niveles de resiliencia en los docentes son, a su vez, detectados por el instrumento. Esta medida sería un elemento más que permita a los agentes educativos 
hacer ajustes para mejorar y/o fortalecer la calidad en la formación de los docentes y en la educación impartida en las escuelas.

- El contar con un instrumento regional permitirá estimar con mayor certeza los niveles de resiliencia, sin la debilidad de los instrumentos creados para otros ambientes culturales. Con la ER-D el educador dispone de un instrumento que le ayudará en la labor de autoconocimiento y en reorientar su actitud frente a su desempeño como profesional. La ER-D ofrece una posibilidad real de ser utilizada en la investigación e intervención psicoeducativa, focalizando la acción y permitiendo evaluar con mayor precisión el impacto.

- Finalmente, se sugiere aplicar la ER-D en otras localidades, a fin de lograr diagnósticos distritales o regionales utilizables en los planes de los gobiernos sub nacionales en el campo de la educación.

\section{Referencias}

Escurra, L. (1988). Cuantificación de la validez de contenido por criterio de jueces. Revista de Psicología., 6 , 103-111.

Henderson, N. y Milstein, M. (2003). Resiliencia en la escuela. México: Paidós.

Kline, P. (1993). The handbook of the psychological testing. London: Routhledge.

Morales, P. (2006). Medición de actitudes en psicología y educación: construcción de escalas y problemas metodológicos. Madrid: Universidad Pontificia Comillas.

Muñiz, J. (1996). Psicometría. Madrid: Universitas.

Ospina D. E. (2007). La medición de la resiliencia. Investigación y Educación en Enfermería. (25) 1. 58-65. Extraído el 24 de Enero de 2009 desde: http://www.scielo.org.co/scielo.php?pid=S012053072007000100006\&script=sci_arttext

UMC. (2002). El programa internacional para la evaluación de estudiantes de la OCDE (PISA) y la participación del Perú. Boletín UMC $N^{o} 21$. Lima: MINEDU.

UNESCO. (1998). Primer Estudio Internacional Comparativo. Santiago de Chile: UNESCO - LLECE. 
Anexo A.

\begin{tabular}{|l|l|l|l|l|}
\hline $\begin{array}{l}\text { Totalmente de } \\
\text { acuerdo }\end{array}$ & De Acuerdo & Indeciso & En desacuerdo & $\begin{array}{l}\text { Totalmente en des- } \\
\text { acuerdo }\end{array}$ \\
\hline 5 & 4 & 3 & 2 & 1 \\
\hline
\end{tabular}

\begin{tabular}{|c|c|c|}
\hline $\mathrm{N}^{\circ}$ & ITEM & RESPUESTA \\
\hline 1 & Me cuesta aceptar las sugerencias de los otros. & $\begin{array}{lllll}5 & 4 & 3 & 2 & 1\end{array}$ \\
\hline 2 & Conozco cuáles son mis deberes en la institución educativa. & $\begin{array}{lllll}5 & 4 & 3 & 2 & 1\end{array}$ \\
\hline 3 & $\begin{array}{l}\text { Participo en cursos de corta duración programados por el MINEDU } \\
\text { u otras instituciones. }\end{array}$ & $\begin{array}{lllll}5 & 4 & 3 & 2 & 1\end{array}$ \\
\hline 4 & $\begin{array}{l}\text { He recibido alguna felicitación o recompensa que haya aumentado } \\
\text { mi sentido de pertenencia hacia mi institución educativa. }\end{array}$ & $\begin{array}{lllll}5 & 4 & 3 & 2 & 1\end{array}$ \\
\hline 5 & Me siento inseguro de mi capacidad profesional. & $\begin{array}{lllll}5 & 4 & 3 & 2 & 1\end{array}$ \\
\hline 6 & $\begin{array}{l}\text { Me incomoda asumir responsabilidades administrativas que están } \\
\text { fuera de mi carga horaria. }\end{array}$ & $\begin{array}{lllll}5 & 4 & 3 & 2 & 1\end{array}$ \\
\hline 7 & $\begin{array}{l}\text { Estoy dispuesto a interactuar con todos los docentes de mi institu- } \\
\text { ción educativa a pesar de los problemas que puedan surgir. }\end{array}$ & $\begin{array}{lllll}5 & 4 & 3 & 2 & 1\end{array}$ \\
\hline 8 & $\begin{array}{l}\text { Cumplo con la entrega de documentación que me solicita la direc- } \\
\text { ción en el tiempo requerido. }\end{array}$ & $\begin{array}{lllll}5 & 4 & 3 & 2 & 1\end{array}$ \\
\hline 9 & Me capacito en el aprendizaje de otros idiomas. & $\begin{array}{lllll}5 & 4 & 3 & 2 & 1\end{array}$ \\
\hline 10 & $\begin{array}{l}\text { Tengo la percepción de que no soy valorado por mi institución } \\
\text { educativa. }\end{array}$ & $\begin{array}{lllll}5 & 4 & 3 & 2 & 1\end{array}$ \\
\hline 11 & $\begin{array}{l}\text { Creo que puedo alcanzar mis objetivos profesionales dentro de mi } \\
\text { institución educativa. }\end{array}$ & $\begin{array}{lllll}5 & 4 & 3 & 2 & 1\end{array}$ \\
\hline 12 & $\begin{array}{l}\text { Asumo de buen grado responsabilidades extracurriculares, que están } \\
\text { fuera de mi carga horaria. }\end{array}$ & $\begin{array}{lllll}5 & 4 & 3 & 2 & 1\end{array}$ \\
\hline 13 & $\begin{array}{l}\text { Dialogo con frecuencia con los docentes, alumnos y padres de fami- } \\
\text { lia de mi institución educativa. }\end{array}$ & $\begin{array}{lllll}5 & 4 & 3 & 2 & 1\end{array}$ \\
\hline 14 & Frecuentemente me sancionan por incumplir con mis obligaciones. & $\begin{array}{lllll}5 & 4 & 3 & 2 & 1 \\
\end{array}$ \\
\hline 15 & $\begin{array}{l}\text { Asisto a cursos de capacitación referidos a mejorar mi desempeño } \\
\text { profesional. }\end{array}$ & $\begin{array}{lllll}5 & 4 & 3 & 2 & 1\end{array}$ \\
\hline 16 & Me siento ajeno a mi institución educativa. & $\begin{array}{lllll}5 & 4 & 3 & 2 & 1\end{array}$ \\
\hline 17 & $\begin{array}{l}\text { Creo que obstaculizo el alcanzar la misión y objetivos de mi institu- } \\
\text { ción educativa. }\end{array}$ & $\begin{array}{lllll}5 & 4 & 3 & 2 & 1\end{array}$ \\
\hline 18 & $\begin{array}{l}\text { Asumo de buen grado responsabilidades tutoriales, que están fuera } \\
\text { de mi carga horaria ya sea con mis alumnos o padres de familia. }\end{array}$ & $\begin{array}{lllll}5 & 4 & 3 & 2 & 1\end{array}$ \\
\hline 19 & $\begin{array}{l}\text { Casi no participo en reuniones sociales, de camaraderías, paseos u } \\
\text { otros con los demás docentes de mi institución. }\end{array}$ & $\begin{array}{lllll}5 & 4 & 3 & 2 & 1\end{array}$ \\
\hline 20 & $\begin{array}{l}\text { Acepto de buen grado los procedimientos administrativos que se } \\
\text { solicitan en mi institución educativa (justificaciones, uso de equipos, } \\
\text { solicitudes). }\end{array}$ & $\begin{array}{lllll}5 & 4 & 3 & 2 & 1\end{array}$ \\
\hline
\end{tabular}




\begin{tabular}{|c|c|c|}
\hline 21 & Tengo una buena opinión de mi desempeño profesional. & $\begin{array}{lllll}5 & 4 & 3 & 2 & 1\end{array}$ \\
\hline 22 & $\begin{array}{l}\text { Las actividades que propongo no suelen ser escuchadas por la comu- } \\
\text { nidad educativa. }\end{array}$ & $\begin{array}{lllll}5 & 4 & 3 & 2 & 1\end{array}$ \\
\hline 23 & $\begin{array}{l}\text { Mis colegas poseen potencial que garantice la excelencia en la labor } \\
\text { educativa. }\end{array}$ & $\begin{array}{lllll}5 & 4 & 3 & 2 & 1\end{array}$ \\
\hline 24 & No acepto las propuestas de participación cuando el colegio me lo sugiere. & $\begin{array}{lllll}5 & 4 & 3 & 2 & 1\end{array}$ \\
\hline 25 & Interactúo fácilmente con todos los miembros de la institución educativa. & $\begin{array}{lllll}5 & 4 & 3 & 2 & 1 \\
\end{array}$ \\
\hline 26 & $\begin{array}{l}\text { Casi no participo en la elaboración del reglamento interno de mi } \\
\text { institución educativa. }\end{array}$ & $\begin{array}{lllll}5 & 4 & 3 & 2 & 1\end{array}$ \\
\hline 27 & Me siento orgulloso de mi trabajo y de lo que estudio. & $\begin{array}{lllll}5 & 4 & 3 & 2 & 1 \\
\end{array}$ \\
\hline 28 & Expreso mensajes de valoración y apoyo a mis colegas. & $\begin{array}{lllll}5 & 4 & 3 & 2 & 1\end{array}$ \\
\hline 29 & Siento que mi desempeño en la escuela es valorado por los demás. & $\begin{array}{lllll}5 & 4 & 3 & 2 & 1 \\
\end{array}$ \\
\hline 30 & $\begin{array}{l}\text { Aprovecho el tiempo que la institución brinda para capacitaciones, } \\
\text { jornadas pedagógicas y otras dentro o fuera del horario de trabajo. }\end{array}$ & $\begin{array}{lllll}5 & 4 & 3 & 2 & 1\end{array}$ \\
\hline 31 & $\begin{array}{l}\text { Me resulta difícil dialogar y/o trabajar con las personas que ocupan } \\
\text { cargos superiores en la institución educativa. }\end{array}$ & $\begin{array}{lllll}5 & 4 & 3 & 2 & 1\end{array}$ \\
\hline 32 & $\begin{array}{l}\text { Colaboro para que las reglas y políticas de mi institución educativa } \\
\text { sean mejoradas y entendidas por todos los miembros. }\end{array}$ & $\begin{array}{lllll}5 & 4 & 3 & 2 & 1\end{array}$ \\
\hline 33 & Creo que soy bueno en mi trabajo. & $\begin{array}{lllll}5 & 4 & 3 & 2 & 1\end{array}$ \\
\hline 34 & No suelo recibir ningún aliciente en mi trabajo. & $\begin{array}{lllll}5 & 4 & 3 & 2 & 1 \\
\end{array}$ \\
\hline 35 & Siento que mi esfuerzo por enseñar es valorado por mis alumnos. & $\begin{array}{lllll}5 & 4 & 3 & 2 & 1\end{array}$ \\
\hline 36 & $\begin{array}{l}\text { Participo en las capacitaciones o jornadas de trabajo que la institu- } \\
\text { ción ha programado. }\end{array}$ & $\begin{array}{lllll}5 & 4 & 3 & 2 & 1\end{array}$ \\
\hline 37 & $\begin{array}{l}\text { Me resulta fácil dialogar y/o trabajar con las personas que ocupan } \\
\text { cargos de menor jerarquía en la institución educativa. }\end{array}$ & $\begin{array}{lllll}5 & 4 & 3 & 2 & 1\end{array}$ \\
\hline 38 & $\begin{array}{l}\text { Cuando no estoy de acuerdo con algunas reglas de mi institución } \\
\text { educativa, intervengo sugiriendo su cambio. }\end{array}$ & $\begin{array}{lllll}5 & 4 & 3 & 2 & 1\end{array}$ \\
\hline 39 & Estoy satisfecho con lo que he logrado en mi vida profesional hasta ahora. & $\begin{array}{lllll}5 & 4 & 3 & 2 & 1\end{array}$ \\
\hline 40 & $\begin{array}{l}\text { Animo o ayudo a mis colegas a tomar iniciativas para bien de ellos o } \\
\text { de la institución educativa. }\end{array}$ & $\begin{array}{lllll}5 & 4 & 3 & 2 & 1\end{array}$ \\
\hline 41 & $\begin{array}{l}\text { Siento que los directivos valoran mi profesión de maestro de escuela } \\
\text { pública. }\end{array}$ & $\begin{array}{lllll}5 & 4 & 3 & 2 & 1\end{array}$ \\
\hline 42 & Percibo que las capacitaciones me sirven para adquirir nuevas habilidades. & $\begin{array}{lllll}5 & 4 & 3 & 2 & 1 \\
\end{array}$ \\
\hline 43 & $\begin{array}{l}\text { Hago distinciones en el trato con los otros, de acuerdo a la jerarquía } \\
\text { que ocupan. }\end{array}$ & $\begin{array}{lllll}5 & 4 & 3 & 2 & 1\end{array}$ \\
\hline 44 & He adquirido nuevas habilidades en el transcurso de mi labor docente. & $\begin{array}{lllll}5 & 4 & 3 & 2 & 1\end{array}$ \\
\hline 45 & $\begin{array}{l}\text { Ayudo a mis compañeros docentes que pudieran presentar algunas } \\
\text { dificultades en su vida personal y profesional. }\end{array}$ & $\begin{array}{lllll}5 & 4 & 3 & 2 & 1\end{array}$ \\
\hline 46 & $\begin{array}{l}\text { La institución educativa suele apoyar las iniciativas de los docentes } \\
\text { dentro y fuera del aula. }\end{array}$ & $\begin{array}{lllll}5 & 4 & 3 & 2 & 1\end{array}$ \\
\hline
\end{tabular}




\begin{tabular}{|c|c|c|}
\hline 47 & $\begin{array}{l}\text { La institución educativa no me concede el tiempo suficiente para } \\
\text { realizar las actividades de enseñanza-aprendizaje con los alumnos. }\end{array}$ & $\begin{array}{lllll}5 & 4 & 3 & 2 & 1\end{array}$ \\
\hline 48 & $\begin{array}{l}\text { Pienso que no poseo suficientes habilidades para participar con } \\
\text { eficacia en la escuela. }\end{array}$ & $\begin{array}{lllll}5 & 4 & 3 & 2 & 1\end{array}$ \\
\hline 49 & $\begin{array}{l}\text { Me disgusta planificar mis unidades didácticas con otros docentes de } \\
\text { mi área o nivel. }\end{array}$ & $\begin{array}{lllll}5 & 4 & 3 & 2 & 1\end{array}$ \\
\hline 50 & $\begin{array}{l}\text { Soy inconstante a la hora de planificar y ejecutar las sesiones de } \\
\text { enseñanza-aprendizaje dentro y fuera del aula. }\end{array}$ & $\begin{array}{lllll}5 & 4 & 3 & 2 & 1\end{array}$ \\
\hline 51 & Me cuesta recibir ayuda de otros cuando tengo alguna dificultad. & $\begin{array}{lllll}5 & 4 & 3 & 2 & 1 \\
\end{array}$ \\
\hline 52 & $\begin{array}{l}\text { Creo que es innecesario que se recompense todas las iniciativas } \\
\text { educativas individuales. }\end{array}$ & $\begin{array}{lllll}5 & 4 & 3 & 2 & 1\end{array}$ \\
\hline 53 & $\begin{array}{l}\text { Siento que los directivos de la institución no apoyan las propuestas } \\
\text { innovadoras que puedo aportar. }\end{array}$ & $\begin{array}{lllll}5 & 4 & 3 & 2 & 1\end{array}$ \\
\hline 54 & Estoy convencido que para aprender es necesario dedicarle tiempo. & $\begin{array}{lllll}5 & 4 & 3 & 2 & 1 \\
\end{array}$ \\
\hline 55 & $\begin{array}{l}\text { Puedo trabajar en equipo con todos los miembros de mi institución } \\
\text { educativa. }\end{array}$ & $\begin{array}{lllll}5 & 4 & 3 & 2 & 1\end{array}$ \\
\hline 56 & $\begin{array}{l}\text { Amplío el currículo de estudio mediante la implementación de pro- } \\
\text { yectos, módulos, innovaciones, investigaciones. }\end{array}$ & $\begin{array}{lllll}5 & 4 & 3 & 2 & 1\end{array}$ \\
\hline 57 & $\begin{array}{l}\text { Me es difícil escuchar las sugerencias que otros puedan hacerme } \\
\text { sobre mi desempeño profesional. }\end{array}$ & $\begin{array}{lllll}5 & 4 & 3 & 2 & 1\end{array}$ \\
\hline 58 & $\begin{array}{l}\text { Pienso que el personal recibe incentivos, reconocimientos y gratifica- } \\
\text { ciones cuando lo merece por parte de la institución educativa. }\end{array}$ & $\begin{array}{lllll}5 & 4 & 3 & 2 & 1\end{array}$ \\
\hline 59 & Estoy satisfecho con la remuneración que percibo. & $\begin{array}{lllll}5 & 4 & 3 & 2 & 1 \\
\end{array}$ \\
\hline 60 & Siento que los éxitos de la escuela no son mis éxitos. & $\begin{array}{lllll}5 & 4 & 3 & 2 & 1 \\
\end{array}$ \\
\hline 61 & $\begin{array}{l}\text { Trabajo en equipo en las comisiones que se me asignan en la institu- } \\
\text { ción educativa. }\end{array}$ & $\begin{array}{lllll}5 & 4 & 3 & 2 & 1\end{array}$ \\
\hline 62 & $\begin{array}{l}\text { Me involucro apasionadamente en los proyectos que mi escuela } \\
\text { planifica y ejecuta. }\end{array}$ & $\begin{array}{lllll}5 & 4 & 3 & 2 & 1\end{array}$ \\
\hline 63 & Desconozco lo que acontece en mi institución. & $\begin{array}{lllll}5 & 4 & 3 & 2 & 1 \\
\end{array}$ \\
\hline 64 & $\begin{array}{l}\text { Fomento la formación de círculos de estudio para el mejoramiento } \\
\text { del desempeño profesional de los docentes de mi institución educati- } \\
\text { va (seminarios, talleres, capacitaciones). }\end{array}$ & $\begin{array}{lllll}5 & 4 & 3 & 2 & 1\end{array}$ \\
\hline 65 & Me intimidan los retos y las oportunidades existentes en mi escuela. & $\begin{array}{lllll}5 & 4 & 3 & 2 & 1 \\
\end{array}$ \\
\hline 66 & Considero que soy parte de la consecución de logros en la escuela. & $\begin{array}{lllll}5 & 4 & 3 & 2 & 1 \\
\end{array}$ \\
\hline 67 & Siento que merezco un mejor sueldo para el trabajo que realizo. & $\begin{array}{lllll}5 & 4 & 3 & 2 & 1\end{array}$ \\
\hline 68 & $\begin{array}{l}\text { Me gustaría conocer más los requerimientos de la escuela para poder } \\
\text { aportar con un trabajo más significativo. }\end{array}$ & $\begin{array}{lllll}5 & 4 & 3 & 2 & 1\end{array}$ \\
\hline 69 & $\begin{array}{l}\text { Participo en actividades estimulantes y significativas en mi trabajo } \\
\text { para el desarrollo de la escuela. }\end{array}$ & $\begin{array}{lllll}5 & 4 & 3 & 2 & 1\end{array}$ \\
\hline
\end{tabular}

